\title{
Psychoacoustic Measures and Speech Perception of Compressed Rate of Sentences in Noise in Individuals with Congenital and Acquired Blindness
}

\author{
Jhanja Pratihari ${ }^{1}$ \\ ${ }^{1}$ Department of Audiology and Speech and Language Pathology, \\ Samvaad Institute of Speech and Hearing, Bengaluru, Karnataka, India.
}

\section{ABSTRACT}

\section{BACKGROUND}

Without visual information, blind speakers rely solely on the auditory signal to recover phonological information. We wanted to measure the psychoacoustic measures and perception of compressed speech in noise in individuals with congenital and acquired blindness and correlate the temporal resolution and frequency resolution ability with individuals with normal sight.

\section{METHODS}

The clinical group (age range-12-20 years) contains two subgroups- congenital blindness and acquired blindness, with normal hearing each containing 15 participants. 15 participants with normal vision were selected as controls. To measure the temporal processing ability each participant was asked to perform Gap detection test. Similarly, difference limen frequency was estimated from pitch discrimination of pure tones. The task for speech perception of compressed sentences was done at different rate of compression at different SNRs.

\section{RESULTS}

Results indicated that there was significant difference in gap detection threshold between congenital blinds and normal sighted participants as well as between acquired blinds and normal sighted participants. There was significant difference in frequency discrimination scores between blind listeners and normal sighted participants. The sentence perception score was better in those participants who have congenital blindness than acquired blindness followed by normal sighted participants in each experimental condition.

\section{CONCLUSIONS}

From the investigation, it can be concluded that the psychoacoustic measures are better in individuals with blindness than individuals with normal sight. speech perception deteriorates with an increase in noise in both normal sighted individuals and individuals with blindness. Effect of noise is more for individuals with normal sight than blind listeners.

\section{KEY WORDS}

Congenital Blindness, Acquired Blindness, Psychoacoustic Measures, Speech Perception
Corresponding Author:

Jhanja Pratihari,

430, United Ellysium,

Seegehalli, Bangalore-560067,

Karnataka, India.

E-mail: jhanja.pratihari690@gmail.com

DOI: 10.14260/jemds/2020/163

Financial or Other Competing Interests: None.

How to Cite This Article:

Pratihari J. Psychoacoustic Measures and Speech Perception of Compressed Rate of Sentences in Noise in Individuals with Congenital and Acquired Blindness. J. Evolution Med. Dent. Sci. 2020;9(10):749752, DOI: 10.14260/jemds/2020/163

Submission 03-01-2019,

Peer Review 12-02-2020,

Acceptance 19-02-2020,

Published 09-03-2020.

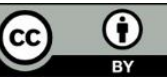




\section{BACKGROUND}

Without visual information, blind speakers rely solely on the auditory signal to recover phonological information. In a review of studies conducted on blind and sighted speakers, researchers ${ }^{1}$ showed that studies have produced somewhat contradictory results regarding auditory acuity in blind and sighted subjects. Researchers ${ }^{2}$ studied rhythm perception and frequency discrimination in music and speech in clear and distorted background noise or reduced tempo conditions in 30 young speakers between 10 and 15 years of age belonging to three groups: totally blind, partially blind, and sighted. Blind speakers performed better than sighted speakers in frequency discrimination tasks, but no difference was found between the two groups in speech identification tasks in distorted conditions. Researchers $3 \& 4$ also conducted a series of perceptual experiments on blind and sighted adults. They found no difference in frequency discrimination thresholds, intensity discrimination thresholds, or duration discrimination thresholds. Researchers 5,6 \& 7 also found superior non-speech auditory perceptual abilities in blind speakers. However, it should be noted that the blind speaker groups in these studies were heterogeneous in many respects, for instance, concerning age, age at blindness, degree of blindness, etc. Such variability is confounded with visual impairment and could have greatly influenced the results. Hence in the present study three age matched groups viz., congenital blind, acquired blind and normal sighted individuals participated in temporal processing and speech perception tasks. So, in this investigation we measure the psychoacoustic measures and speech perception of compressed speech in noise in different group of subjects.

\section{METHODS}

This is a cross-sectional study conducted among 45 participants aged between 12 - 20 years with normal hearing sensitivity, normal middle ear function and right-handed Kannada speaker. The sample size has been approved by the institutional Ethical committee. The participants selected were divided into two groups, the clinical and the control group. Those with normal vision were included under control group and individuals with visual impairment belonged to the clinical group. The clinical group of 30 participants was divided into 2 subgroups each containing 15 participants. Subgroup I includes individuals with congenital blindness and sub group II includes individuals with acquired blindness. The control group includes individuals with normal vision.

Our current investigation includes a routine audiological evaluation along with the current test procedures which include-

1. A detailed case history.

2. The modified version of Hughson and Westlake procedure (Carhart and Jerger, 1959) was used to obtain air conduction and bone conduction thresholds at octave frequencies between $250 \mathrm{~Hz}$ to $8000 \mathrm{~Hz}$ and $250 \mathrm{~Hz}$ to $4000 \mathrm{~Hz}$ respectively.

3. As a part of speech audiometry, speech recognition scores were obtained with spondee word list that was given by
Vandana (1998). Uncomfortable level (UCL) for running speech was also obtained.

4. MCL was obtained using Shapiro's procedure.

5. Immittance audiometry was carried out by varying the pressure from $+200 \mathrm{daPa}$ to $-400 \mathrm{daPa}$ for a probe tone frequency of $226 \mathrm{~Hz}$. Ipsilateral and contralateral stapedial acoustic reflexes thresholds were measured for $500 \mathrm{~Hz}, 1000 \mathrm{~Hz}, 2000 \mathrm{~Hz}$, and $4000 \mathrm{~Hz}$ pure tones.

6. Temporal processing ability from each of the study participant was obtained from the Gap Detection Test (GDT). Maximum likelihood procedure (MLP) m-code stored in MATLAB (2009B) was used to deliver the gap in three interval force choice method.

7. Difference limen frequency was estimated from pitch discrimination of pure tones. (frequency discrimination test). Maximum likelihood procedure (MLP) m-code stored in MATLAB (2009B) was used to deliver the tones of different frequencies in three alterative force choice (AFC) method.

8. A total of six sentence lists having different rate of compression $(30 \%$ and $35 \%)$ and three lists of unprocessed sentences at different SNRs (quite, $0 \mathrm{~dB}$, $1 \mathrm{~dB},-3 \mathrm{~dB}$ ) were stored in personal laptop. These lists were delivered through audiometer and the output of it was presented in headphone at subject's MCL. These lists were randomized and presented at listener's comfortable level. The participants were instructed to repeat the sentence heard. Further, the succeeding sentences were presented once after repeating the sentence heard.

\section{Statistical Analysis}

Descriptive statistical analyses were done to compute the mean and standard deviation distribution of results of gap detection test \&amp; frequency discrimination test for the three groups. A one-way analysis of variance was performed to examine the effect of gap detection threshold on groups. Post hoc comparison using the Fisher LSD test was applied to compare the results of gap detection test between each group. MANOVA was conducted to know the effect of groups on frequency discrimination at each frequency. Duncan post hoc test was conducted to know in which groups have caused significant differences on frequency discrimination at each frequency.

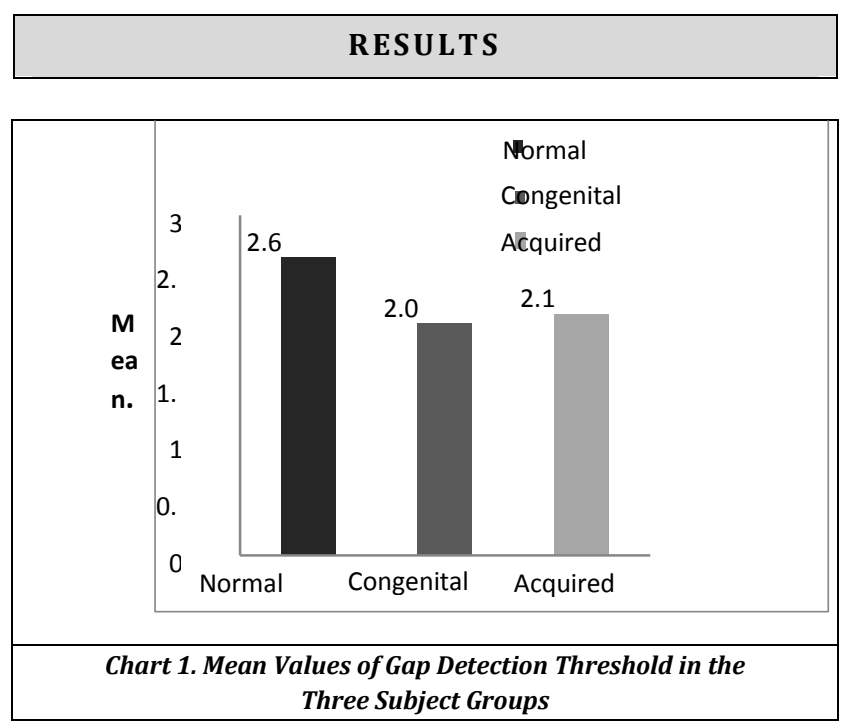



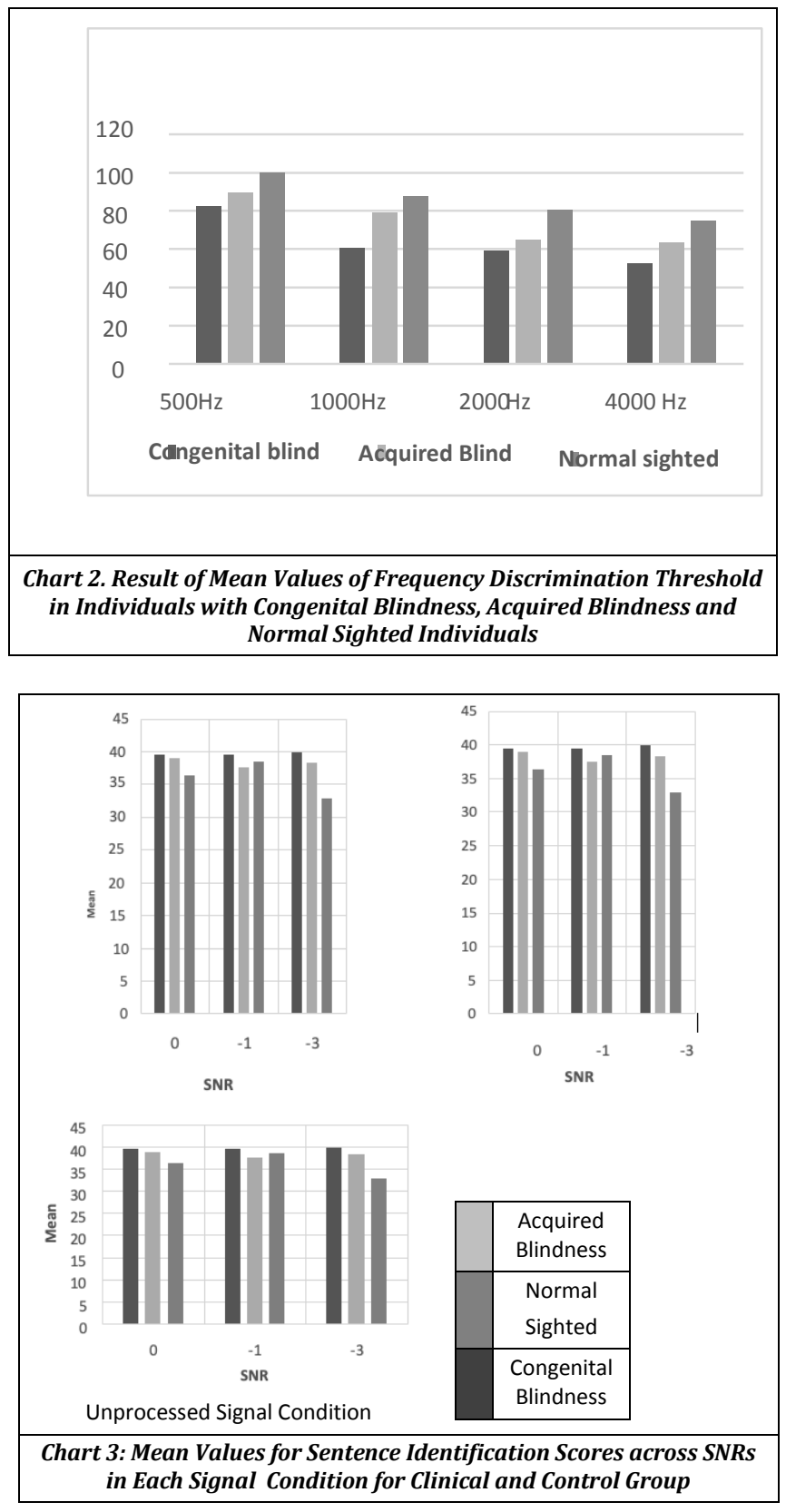

Normal sighted participants showed a significant higher gap detection threshold than congenital or acquired blind participants. There was significant difference in gap detection threshold between congenital blinds and normal sighted participants as well as between acquired blinds and normal sighted participants. But there was no significant difference between congenital and acquired blinds. Frequency discrimination score (FDS) on $500 \mathrm{~Hz}$ was lesser in congenital blind group than acquired blind group followed by normal sighted group. There was a significant difference on frequency discrimination scores between groups at each frequency. There was significant difference in frequency discrimination scores between congenital blinds and normal sighted participants as well as between acquired blinds and normal sighted participants. But there was no significant difference between congenital and acquired blinds. The sentence perception score was better in those participants who have congenital blindness than acquired blindness followed by normal sighted participants in each experimental condition. The perception of sentences was better in unprocessed experimental condition than $30 \%$ compressed sentence followed by $35 \%$ compressed perception of sentences.

Sentence perception was poorer in $-3 \mathrm{~dB}$ SNR than $-1 \mathrm{~dB}$ SNR followed by $0 \mathrm{~dB}$ SNR in all the three experimental conditions. (unprocessed, 30\% compression, 35\% compression). There was a significant difference between groups on sentence perception. There is no effect of rate in each group. There was significant difference in between congenital blinds and normal sighted participants as well as between acquired blinds and normal sighted participants. But there is no significant difference between congenital and acquired blinds on speech perception within each experimental condition in all SNRs.

\section{DISCUSSION}

Comparison of Gap Detection and Frequency Discrimination Scores between the Groups

The reason of better perception in blinds could be best temporal and frequency processing abilities than sighted individuals. This was ascertained in the present study by administering gap detection test and frequency discrimination test at frequencies $500 \mathrm{~Hz}$ to $4000 \mathrm{~Hz}$ (in octave). The results revealed that congenital and acquired blind individuals were performed significantly better on scores of GDT and FDT than normal sighted participants. As expected, FDT was smaller at high frequency than at low frequency. This was true in each group. However, blind individuals took relatively smaller difference in frequencies in discriminating two tones than sighted subjects. The possible reason for obtaining low gap detection threshold and superiority on FDT test in blind individuals could be filtering characteristics of the peripheral auditory system. That is for any given signal (speech and non-speech signal) their auditory system analyses subtle temporal pattern in each activated auditory filters.

\section{Effect of Compression Rate and Noise on Sentence Identification}

At each SNR, the mean perception for $35 \%$ compressed rate of sentence was lesser than $30 \%$ compressed rate of sentence followed by unprocessed sentences. However, these differences did not reach significant. This is because with rate change in sentence, a gap between phonemes reduces such that auditory system should quickly process to these rapidly changing information to interpret an inherent meaning. In addition, sentence perception reduced at low SNR, irrespective of rate change in sentence. In the presence of noise, there is high chance of alteration in inherent spectral and temporal cues, which provides additional load on auditory system to infer the meaning of sentence. This exacerbates on perception with change in rate of sentence at low SNRs. Possible reason could be gap between phonemes reduces and acoustic features are swiftly changed. Further fills an available gap between phonemes and also pauses in sentences. This challenges the auditory system to process the information sent at different levels of auditory pathway. At -3 $\mathrm{dB}$ SNR, sentence perception was poorer than $-1 \mathrm{~dB}$ SNR 
followed by $0 \mathrm{~dB}$ SNR. This phenomenon was observed in study participants of each group.

\section{Comparison of Sentence Perception between the Groups}

Interesting finding was mean sentence perception score was higher in congenital blind group than acquired blind group then followed by normal hearing group. This was observed in each experimental condition and in each SNR. It is in accordance with the research reports of researchers ${ }^{8}$ who demonstrated blind people can comprehend speech sped up to 22 syllables per second which was found to be way high than normal sighted individuals who comprehend speech sped up to 8 syllables per second. It means their auditory processing mechanism responds twice faster than normal sight individuals. According to the studies, blind people are highly proficient in ultra-fast speech perception as shown by significant hemodynamic activation of right hemispheric primary visual cortex, contralateral fusiform gyrus, and bilateral pulvinar.

In addition, subtle temporal patterns are compared between filters. These processes at peripheral structures are happened in all the study participants. But superiority in these processing occurs in blind individuals than normal sighted individuals. Further, these peripheral processing is successively relied at larger areas of central structure in blind participants than normal sighted participants. To support the speculation a study conducted by researchers ${ }^{9}$ reported that auditory cortical areas are strongly represents subtle cues in auditory cues in blind individuals, which were confirmed by functional magnetic resonance. In addition, due to visual loss more attention is devoted in processing auditory cues. This causes blind individuals attend to subtle cues in auditory stimuli. It was also reported that part of cerebral cortical structure that normally responds to vision was responded to a speech stimulus. Our finding reinforces the belief that blind children develop their hearing ability better to compensate the loss of vision.

\section{CONCLUSIONS}

From the investigation, it can be concluded that speech perception deteriorates with an increase in noise in both normal sighted individuals and individuals with blindness. Effect of noise is more for individuals with normal sight than blind listeners. Speech perception is better in unprocessed sentences than compressed rate of sentences. But there is no effect of change in compression rate in all the groups. The gap detection and frequency discrimination ability is also better in both groups ( congenital blindness and acquired blindness) than in normal sighted individuals.

\section{REFERENCES}

[1] Miller L. Diderot reconsidered: visual impairment and auditory compensation. Journal of Visual Impairment \& Blindness 1992;86(5):206-10.

[2] Stankov L, Spilsbury G. The measurement of auditory abilities of blind, partially sighted, sighted children. Applied Psychological Measurement 1978;2(4):491-503.

[3] Niemeyer W, Starlinger I. Do the blind hear better? Investigations on auditory processing in congenital or early acquired blindness. II. Central functions. Audiology 1981;20(6):510-15.

[4] Starlinger I, Niemeyer W. Do the blind hear better? Investigations on auditory processing in congenital or early acquired blindness. I. Peripheral functions. Audiology 1981;20(6):503-9.

[5] Lucas SA. Auditory discrimination and speech production in the blind child. International Journal of Rehabilitation Research 1984;7(1):74-6.

[6] Hugdahl K, Ek M, Takio F, et al. Blind individuals show enhanced perceptual and attentional sensitivity for identification of speech sounds. Brain Research Cognitive Brain Research 2004;19(1):28-32.

[7] Gougoux F, Lepore F, Lassonde $M$, et al. Neuropsychology: pitch discrimination in the early blind. Nature 2004;430(6997):309.

[8] Collignon 0, Dormal G, Albouy G, et al. Impact of blindness onset on the functional organization and the connectivity of the occipital cortex. Brain 2013;136(Pt 9):2769-83.

[9] Stevens AA, Weaver KE. Functional characteristics of auditory cortex in the blind. Behavioral Brain research 2009;196(1):134-8. 